\title{
Exponential groups 2: Extensions of centralizers and tensor completion of CSA-groups
}

\author{
by \\ Myasnikov A.G., Remeslennikov V.N.
}

This is the second article in the series of papers by the authors on the theory of exponential groups. In the first one [15] we discussed foundations of this theory. Definitions necessary for independent understanding of the present article are given in the introduction and the first section. The theory of exponential groups begins with results of A.Mal'cev [11], P.Hall [0], G.Baumslag [4] and R.Lyndon [9]. The axiomatic notion of an exponential group was introduced by R.Lyndon (1960). In [13] a new axiom was added to Lyndon's definition to obtain a new notion of an exponential group. The refined version is more convenient because it coincides exactly with the notion of a module over a ring in the abelian case, whereas abelian exponential groups after Lyndon provide a far wider class.

Recall the main definition from [13].

Let $A$ be an arbitrary associative ring with identity and $G$ a group. Fix an action of the ring $A$ on $G$, i.e. a map $G \times A \rightarrow G$. The result of the action of $\alpha \in A$ on $g \in G$ is written as $g^{\alpha}$. Consider the following axioms:

1. $g^{1}=g, g^{0}=1,1^{\alpha}=1$;

2. $g^{\alpha+\beta}=g^{\alpha} \cdot g^{\beta}, g^{\alpha \beta}=\left(g^{\alpha}\right)^{\beta}$;

3. $\left(h^{-1} g h\right)^{\alpha}=h^{-1} g^{\alpha} h$;

4. $[g, h]=1 \Longrightarrow(g h)^{\alpha}=g^{\alpha} h^{\alpha}$.

Definition 1 Groups with axioms 1)-4) are called A-groups.

As usual, one can introduce the notions of $A$-torsion, finitely $A$-generated group, $A$-homomorphism, $A$-isomorphism, and so on.

Our interest to the theory of exponential groups is motivated by the following circumstances. First, many natural classes of groups are $A$-groups. 
For example, unipotent groups over a field $k$ of zero characteristic are $k^{-}$ groups, pro- $p$-groups are exponential groups over the ring of $p$-adic integers, etc. (see [15] for examples). Second, the notion of an $A$-group is a natural generalization of the notion of a module over a ring in the category of noncommutative groups. Third, this notions allows one to define for an arbitrary group $G$ its largest ring of scalars $A(G)$, over which $G$ is an $A(G)$-group. The last notion is an exact analog of the notion of the centroid for rings and algebras (see [13] for details) and plays an important part in many questions. Finally, the notion of an $A$-exponential group is helpful in investigation of model-theoretic problems for noncommutative groups [14]. Let us illustrate this on example of universally free group, i.e. groups that has the same universal theory as a nonabelian free group (see [17] for details). The study of these groups plays a key part in creating model theory of free groups. Here we formulate a principal problem for universally free groups.

Conjecture Every finitely generated universally free group is a subgroup of a free $\mathbf{Z}[x]$-group, where $\mathbf{Z}[x]$ is the ring of polynomials in an indeterminate $x$ with integer coefficients.

This problem requires a detailed study of $\mathbf{Z}[x]$-free groups and their subgroups.

The present article will be devoted to the general theory of tensor $A$ completions of groups with an emphasis on $A$-free groups..

G.Baumslag was the first who introduced the $\mathbf{Q}-$ completion $F^{\mathbf{Q}}$ of a free group $F$ and, what is more important, described it using free products with amalgamation. It allowed him to establish some properties of the group $F^{\mathbf{Q}}$. We develope his approach and apply it for arbitrary rings $A$ of characterisric 0 and groups from a very wide class.

One of the key notions in the theory of $A$-groups is the notion of tensor $A$-completion. The case where a group $G$ is a subgroup of its $A$-completion $G^{A}$ (i.e. $G$ is $A$-faithful) is of a particular interest. In section 2 we prove that the class $\mathcal{F}_{A}$ of all $A$-faithful groups is universally axiomatizable and closed under subgroups, direct products and direct limits, and, in the case when $A$ is an integral domain of characteristic zero, contains all residually torsion-free nilpotent groups. For arbitrary groups it is difficult to give a good constructive description of their $A$-completion. In sections $5-7$ we study the class of CSA-groups, for which a good and concrete description of tensor completion exists. The class of CSA-groups is quite wide, it is universally axiomatizable, 
closed under free products, direct limits and extensions of centralizers (basic facts concerning these constructions are given in sections 3-4); moreover it contains abelian, free, hyperbolic torsion-free groups and groups acting freely on $\Lambda$-trees and universally free groups. For CSA-groups the construction of tensor completion of a group $G$ is obtained as an iterated tree extension of centralizers of the initial group $G$. In particular, any torsion-free CSAgroup is faithful over any ring $A$ with a torsion-free additive group $A^{+}$. In the last section we apply the obtained results to the study of basic properties of $A$-free groups. In particular, canonical and reduced forms of elements in $A$-free groups are introduced, and then commuting and conjugate elements are described. We finish the paper by formulating some open problems on this area.

The results of this article are partially contained in preprints [2, 16].

\section{Tensor completions and free A-groups}

The basic operation in the class of $A$-groups is the operation of tensor completion. Here we give a particular case of this construction (see 15 for the general definition). Later on we always assume that the ring $A$ and its subring $A_{0}$ have a common identity element.

Definition 2 Let $G$ be an $A_{0}$-group and $A_{0}$ a subring of $A$. Then an $A$ group $G^{A}$ is called a tensor $A$-completion of the group $G$ if $G^{A}$ satisfies the following universal property:

1. there exists an $A_{0}$-homomorphism $\lambda: G \rightarrow G^{A}$ such that $\lambda(G) A_{-}$ generates $G^{A}$, i.e. $\langle\lambda(G)\rangle_{A}=G^{A}$

2. for any $A$-group $H$ and an $A_{0}$-homomorphism $\varphi: G \rightarrow H$ there exists the unique $A$-homomorphism $\psi: G^{A} \rightarrow H$ such that $\lambda \circ \psi \varphi$.

If $G$ is an abelian $A_{0}$-group, then the group $G^{A}$ is also abelian, i.e. it is an $A$-module. $G^{A}$ satisfies the same universal property as the tensor product $G \otimes_{A_{0}} A$ of the $A_{0}$-module $G$ and the ring $A$. Therefore $G^{A} \simeq G \otimes_{A_{0}} A$

Theorem 1 [15 Let $G$ be an $A_{0}$-group and $A_{0}$ a subring of $A$. Then there exists a tensor completion $G^{A}$, and it is unique up to an $A$-isomorphism. 
Every group is a $\mathbf{Z}$-group, so one can consider $A$-completions of arbitrary groups for each ring of characteristic zero, i.e. $\mathbf{Z} \leq A$.

Let us formulate the notion of a free $A$-group. Let $A$ be an associative ring with identity, char $A=0$, and $X$ an arbitrary set.

Definition 3 An A-group $F_{A}(X)$ with the set of $A$-generators $X$ is said to be a free $A$-group with base $X$, if for every $A$-group $G$ an arbitrary mapping $\phi_{0}: X \mapsto G$ can be extended to an A-homomorphism $\phi: F_{A}(X) \mapsto G$.

The set $X$ is called the set of free $A$-generators of $F_{A}(X)$. The cardinality of $X$ is called the rank of the group $F_{A}(X)$.

Theorem 2 For every $X$ and $A$ there exists a free $A$-group $F_{A}(X)$; moreover it is unique up to $A$-isomorphism and $F_{A}(X)$ is the tensor A-completion $F(X)^{A}$ of the group $F(X)$.

Let $F(X)$ be a free group in the ordinary sense and let $\phi_{0}: X \mapsto G$ be an arbitrary mapping from $X$ to an $A$-group $G$. Then $\phi_{0}$ induces a homomorphism $\phi_{1}: F(X) \mapsto G$ by a property of a free group. Moreover, the latter mapping induces an $A$-homomorphism $\phi:(F(X))^{A} \mapsto G$. Conseqently, $(F(X))^{A}$ is a free $A$-group with base $X$. Uniqueness follows from the uniqueness of the tensor completion.

\section{Faithful A-completion}

Usually one can construct the $A$-completion of a group $G$ step by step, on each stage creating an extension of a group by defining an action of $A$ on some elements, and then closing the process. Intermediate groups obtained along this construction are partial $A$-groups. Following [15], let us remind the definition of a partial $A$-group.

Definition 4 A group $G$ is a partial A-group iff $g^{\alpha}$ is defined for some $g \in G, \alpha \in A$, and axioms 1)-4) hold wherever the action is defined (this means, in particular, that if one side of equalities 1)-4) is defined, then so is the other side, and they are equal to each other). 
Let $G$ be a partial $A$-group. One can define an $A$-completion of a partial $A$-group $G$ in exactly the same way as in definition 1 (considering $\lambda$ and $\phi$ to be partial $A$-homomorphisms). In particular, if $A_{0}$ is a subring of $A$, then any $A_{0}$-group is also a partial $A$-group. So, consideration of tensor completions of partial $A$-groups covers the case of tensor $A$-completion of $A_{0}$-groups from section 1 .

Definition 5 A partial $A$-group $G$ is called faithful (over $A$ ), if the canonical homomorphism of partial $A$-groups $\lambda: G \longrightarrow G^{A}$ is injective.

Remark 1 1. A partial $A$-group $G$ is faithful iff $G$ is $A$-embeddible into some $A$-group $H$.

2. A group $G$ is faithful over $A$ iff $G$ is residually faithful over $A$.

In [1] it was shown that the operation of tensor completion commutes with the operation of direct product and direct limit:

$$
\left(\oplus G_{i}\right)^{A} \simeq_{A} \oplus G_{i}^{A}, \quad\left(\lim _{\longrightarrow} G_{i}\right)^{A} \simeq_{A} \lim _{\longrightarrow} G_{i}^{A}
$$

Using these statements and the remark above, one can obtain the following propositions:

Propostion 1 Let $\mathcal{F}_{A}$ be a class of all $A$-faithful partial $A$-groups. Then:

1. $\mathcal{F}_{A}$ is closed under direct products;

2. $\mathcal{F}_{A}$ is closed under direct limits.

$\square$ Let $\lambda_{i}: G_{i} \longrightarrow G_{i}^{A}$ be canonical homomorphisms. By the properties above, $\left(\oplus G_{i}\right)^{A} \simeq \oplus G_{i}^{A}$, and if $\lambda=\oplus \lambda_{i}$ is the direct sum of $\lambda_{i}$, then the canonical map $\lambda: \oplus G_{i} \longrightarrow \oplus G_{i}^{A}$ is injective iff each $\lambda_{i}$ is injective. This implies 1$)$.

Let $G=\lim _{\longrightarrow} G_{i}$. Then, as mentioned above, $G^{A}=\lim _{\longrightarrow} G_{i}^{A}$. If $\lambda_{i}: G_{i} \rightarrow G_{i}^{A}$ is the canonical embedding, then there exists a canonical embedding

$$
\lambda: \lim _{\longrightarrow} G_{i} \longrightarrow \lim _{\longrightarrow} G_{i}^{A}=G^{A}
$$

So $G=\lim \longrightarrow G_{i}$ is $A$-faithful.

The following proposition shows that the property of being faithful is a local property. 
Propostion 2 Let $G$ be a partial A-group. Then $G$ is faithful iff every finitely generated subgroup of $G$ is faithful.

Let $\left\{G_{i}, i \in I\right\}$ be the set of all finitely generated partial $A$-subgroups of $G$. Then $G=\lim _{\longrightarrow} G_{i}$, and the statement follows from proposition 1 .

Propostion 3 Let $\mathcal{F}_{A}$ be the class of all faithful partial A-groups. Then:

1. $\mathcal{F}_{A}$ is closed under the operation of taking subgroups;

2. $\mathcal{F}_{A}$ is closed under ultraproducts;

3. $\mathcal{F}_{A}$ is universally axiomatizible.

$\square$ ) is evident by the remark above. To prove 2 ), one can consider instead of the group $G$ the many-sorted structure $\mathcal{A}(G, A)=\left\langle G, G^{A}, A\right\rangle$ with the group operations on $G$ and $G^{A}$, ring operations on $A$, operations of action of $A$ on $G$ (partial) and on $G^{A}$, and the canonical map $\lambda: G \longrightarrow G^{A}$. In $\mathcal{A}(G, A)$ the notion of faithfulness is evidently axiomatizible by one sentence: $g \neq 1 \Longrightarrow \lambda(g) \neq 1$. Instead of the ultraproduct of groups $\Pi G_{i} / D$ over an ultrafilter $D$ one can consider the corresponding ultraproduct $\Pi \mathcal{A}\left(G_{i}, A\right) / D$. By the Scolem Theorem, $\Pi G_{i} / D_{i}$ is a faithful group over the ultrapower $\Pi A / D$ of the ring $A$ over $D$. But there is the diagonal embedding of $A$ into $\Pi A / D$. So $\Pi G_{i} / D$ is a subgroup of the $A$-group $\Pi G_{i}^{A} / D$.

3 ) is a corollary of 1) and 2).

For quite a wide class of rings $A$ we can claim that torsion-free nilpotent groups are faithful over $A$.

Propostion 4 Let $G$ be a torsion-free nilpotent group, and $A$ an integral domain of characteristic 0 . Then $G$ is faithful over $A$.

Let $A^{*}=A \otimes \mathbf{Q}$ be the tensor completion of the ring $A$ over $\mathbf{Q}$. Then $A^{*}$ is a binomial domain (i.e. it contains all binomial coefficients $\left(\begin{array}{l}a \\ n\end{array}\right)$, $a \in A, n \in N)$. It is known [7] that every torsion-free nilpotent group $G$ has an $A^{*}$-completion in the sense of P. Hall. So $G$ is embeddible into an $A^{*}$-group, and hence into an $A$-group. By remark 10 is faithful over $A$.

Theorem 3 Let $G$ be a residually torsion-free nilpotent group. Then $G$ is a faithful group over any integral domain A of characteristic 0. In particularly, free groups, free solvable groups and free polynilpotent groups are faithful over any integral domain of characteristic 0 . 
The first statement follows from proposition 5 and the remark above. Those concrete groups are residually torsion-free nilpotent: see, for example, [10] and [8].

Finally, let us consider some examples of non-faithful groups.

Example 1 Let $G$ be a simple group containing an element of finite order. Then $G$ is not faithful over the field of rational numbers $\mathbf{Q}$. Moreover, $G^{\mathbf{Q}}=1$. Indeed, any $\mathbf{Q}$-group has no elements of finite order, so $G$ is not a subgroup of $G^{\mathbf{Q}}$. But in this case the homomorphism $\lambda: G \longrightarrow G^{\mathbf{Q}}$ has a nontrivial kernel. So the simplicity of $G$ implies that $\lambda(G)=1$, but $G^{\mathbf{Q}}$ is generated by $\lambda(G)$. Therefore, $G^{\mathbf{Q}}=1$.

Example 2 Let $G$ be a torsion-free group with non-unique extraction of roots. Then $G$ is not faithful over $\mathbf{Q}$, since $G^{\mathbf{Q}}$ has unique extraction of roots.

\section{Extensions of centralizers}

In the following we will use a construction of free extension of centralizers of a given group $G$. Let us describe this construction.

Let $G$ be a group. The centralizer of an element $v \in G$ is denoted by $C_{G}(v)$.

If $G=\langle X \mid R\rangle$ is a presentation of $G, Y$ is a set of words in $X$ and $t$ is a new letter (not in $X$ ), then by $\langle G, t \mid[Y, t]=1\rangle$ we will denote the group with the representation $\langle X, t \mid R,[y, t]\rangle_{y \in Y}$.

Now we will define, perhaps, the simplest case of centralizer extension.

Definition 6 The group $G(v, t)=\left\langle G, t \mid\left[C_{G}(v), t\right]=1\right\rangle$ is called the direct of rank 1 extension of the centralizer of the elememt $v$.

It is easy to see that $G(v, t)$ can be obtained from $G$ by an HNN-extension with respect to the identity isomorphism $C_{G}(v) \rightarrow C_{G}(v)$ :

$$
G(v, t)=\left\langle G, t \mid t^{-1} a t=a, a \in C_{G}(v)\right\rangle,
$$

or as a free product with amalgamation:

$$
\left\langle G *\left(C_{G}(v) \times\langle t\rangle\right) \mid C_{G}(v)=C_{G}(v)^{\phi}\right\rangle
$$


with amalgamation by the canonical monomorphism $\phi: C_{G}(v) \rightarrow C_{G}(v) \times\langle t\rangle$.

We will consider also the following much more general construction of free centralizer extensions.

Definition 7 Let $G$ be a group, $C_{G}(v)=C$ the centralizer of an element $v$ from $G$, and $\phi: C \longrightarrow H$ a monomorphism of groups such that $\phi(v) \in Z(H)$. Then the group

$$
G(v, H)=\left\langle G * H \mid C=C^{\phi}\right\rangle
$$

is called an extension of the centralizer $C_{G}(v)$ by the group $H$ with respect to $\phi$.

Some particular cases of this construction are of special interest. We will classify them with respect to the type of the embedding $\phi: C \rightarrow H$. So the extension is central iff $C^{\phi} \leq Z(H)$; direct iff $H=C^{\phi} \times B$; abelian iff $H$ is an abelian group.

Propostion 5 The following statements are true:

1. there exist the canonical embeddings $\lambda_{G}: G \longrightarrow G(v, H)$ and $\lambda_{H}: H \longrightarrow G(v, H)$;

2. $C_{G(v, H)}(v) \geq H$;

3. the group $G(v, H)$ has the following universal property: for any group homomorphisms $\psi_{G}: G \longrightarrow N$ and $\psi_{H}: H \longrightarrow N$ compatible on $C$ and $C^{\phi}$ there exists a unique homomorphism $\psi: G(v, H) \longrightarrow N$ such that the following diagram is commutative:

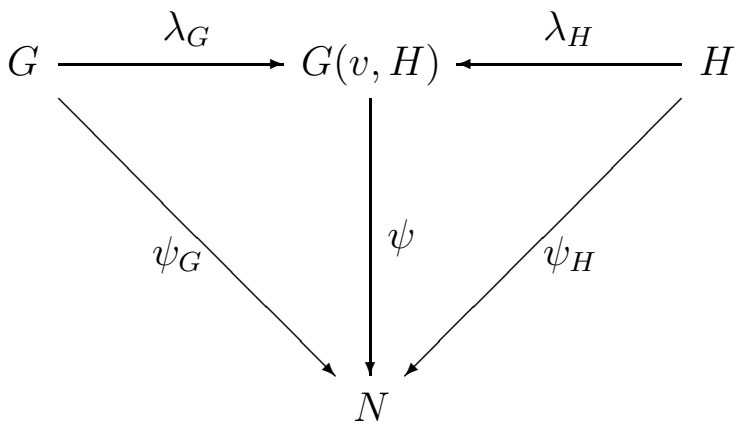


Now we will describe a construction which allows one to extend a set of centralizers at once.

Definition 8 Let $\mathcal{C}=\left\{C_{i}=C_{G}\left(v_{i}\right) \mid i \in I\right\}$ be a set of centralizers in the group $G$. Suppose $\phi_{i}: C_{i} \longrightarrow H_{i}$ is an embedding of $C_{i}$ into $H_{i}$ such that $\phi_{i}(v) \in Z\left(H_{i}\right), i \in I$. We can form a graph of groups



The fundamental group of this graph is called a tree extension of centralizers from $\mathcal{C}$ and is denoted by $G(\mathcal{C}, \mathcal{H}, \Phi)$, where $\mathcal{H}=\left\{H_{i} \mid i \in I\right\}, \Phi=$ $\left\{\phi_{i} \mid i \in I\right\}$.

Again, we will consider central, direct and abelian extensions corresponding to the type of monomorphisms from $\Phi$.

By definition, the group $G(\mathcal{C}, \mathcal{H}, \Phi)$ is the union (direct limit) of the chain of groups

$$
G=G_{0} \hookrightarrow G_{1} \hookrightarrow \cdots \hookrightarrow G_{\alpha} \stackrel{\phi_{\alpha}}{\hookrightarrow} G_{\alpha+1} \hookrightarrow \cdots
$$

where the set of centralizers $\mathcal{C}=\left\{C_{\alpha} \mid \alpha<\lambda\right\}$ is well ordered, $G=$ $G_{0}, G_{\alpha+1}=G_{\alpha}\left(v_{\alpha+1}, H_{\alpha+1}\right), \phi_{\alpha}: G_{\alpha} \longrightarrow G_{\alpha+1}$ is the canonical embedding from proposition 5 , and $G_{\gamma}=\lim _{\longrightarrow} G_{\alpha}$ (here $\alpha<\gamma$ ) for a limit ordinal $\gamma$

Remark 2 The properties of tree extensions of centralizers are similar to those of ordinary extensions of centralizers: there exist the canonical embeddings of $G$ and $H_{i}$ into $G(\mathcal{C}, \mathcal{H}, \Phi)$; the centralizer of $v_{i}$ in $G(\mathcal{C}, \mathcal{H}, \Phi)$ contains the group $H_{i}$ and the group $G(\mathcal{C}, \mathcal{H}, \Phi)$ has the corresponding universal property. Moreover, groups $G(\mathcal{C}, \mathcal{H}, \Phi)$ for different well-orderings have the same universal property, so they are isomorphic. 
Finally, we introduce one more construction: the iterated tree extension of centralizers.

Let $G$ be a group, $\mathcal{C}=\left\{C_{G}\left(v_{i}\right) \mid i \in I\right\}$ a set of centralizers in $G$, and $\Phi=\left\{\phi_{i}: C_{G}\left(v_{i}\right) \rightarrow H_{i}\right\}$ a set of embeddings of these centralizers. Then one can construct the group $G_{1}=G(\mathcal{C}, \mathcal{H}, \Phi)$ and consider again the set of centralizers $\mathcal{C}_{1}$ in $G_{1}$, the set of embeddings $\Phi_{1}$, and the corresponding set of groups $\mathcal{H}_{1}$. As above, the group $G_{2}=G_{1}\left(\mathcal{C}_{1}, \mathcal{H}_{1}, \Phi_{1}\right)$ can be constructed. We can repeat this process using induction on ordinals: if a group $G_{\alpha}$ has been constructed for some ordinal $\alpha$ and the set of centralizers $\mathcal{C}_{\alpha}$ in $G_{\alpha}$, the set of groups $\mathcal{H}_{\alpha}$ and the corresponding set of embeddings $\Phi_{\alpha}$ have been chosen, then

$$
\begin{gathered}
G_{\alpha+1}=G_{\alpha}\left(\mathcal{C}_{\alpha}, \mathcal{H}_{\alpha}, \Phi_{\alpha}\right), \\
G_{\gamma}=\lim _{\overrightarrow{\alpha<\gamma}} G_{\alpha},
\end{gathered}
$$

for a limit ordinal $\gamma$. Thus, for any ordinal $\delta$, starting from a given group $G$, we can obtain the group $G_{\delta}$ by iterating the process above.

Definition 9 The group $G_{\delta}$ is called an iterated tree extension of centralizers up to level $\delta$.

Remark 3 Any iterated tree extension of centralizers can be obtained by transfinite induction using operations of extension of centralizers and direct limits. The group $G$ is embeddible in $G_{\delta}$, and $G_{\delta}$ has a universal property similar to that in proposition 5

\section{Canonical forms of elements in groups which are obtained by abelian extension of cen- tralizers}

In this section we will consider canonical and reduced forms of elements of groups constructed by extensions of centralizers. Of course, there are general theorems about canonical forms in amalgamated free products, but we need some special concrete forms corresponding to this particular situation.

Let $G$ be a group, $v \in G$ an element such that $C_{G}(v)=C$ is a maximal abelian subroup of $G$, and $\phi: C \hookrightarrow A$ an embedding of $C$ into some fixed 
abelian group $A$. In exponential groups it will be convenient to use an exponential writing for elements of $C$ and $A$. Namely, $A \simeq v^{A}=\left\{v^{a} \mid a \in A\right\}$, $C \simeq v^{C}=\left\{v^{c} \mid c \in C\right\}$, in particular, $\langle v\rangle \simeq v^{\mathbf{Z}}=\left\{v^{n} \mid n \in \mathbf{Z}\right\}$. Consider the group $G^{*}$ which is obtained from $G$ by $A$-extension of the centralizer $C$ :

$$
G^{*}=G(v, A)=\left\langle G * v^{A} \mid v^{C}=v^{C^{\phi}}\right\rangle
$$

with respect to the embedding $\phi$.

Denote by $S$ some system of right representatives of $A$ by $C$. Then $v^{S}$ is a system of right representatives of $v^{A}$ by $v^{C}$. Fix any system $\mathcal{R}$ of right representatives of $G$ by $v^{C} \simeq C$. For $g \in G$ we denote by $\bar{g}$ the representative of $g$ in this fixed system of representatives. Clearly, $g=v^{c} \bar{g}$ for some $c \in C$. From general theory of free products with amalgamation we know that every element $g \in G^{*}$ can be written in the form

$$
g=g_{1} v^{a_{1}} \cdots g_{n} v^{a_{n}} g_{n+1}
$$

$a_{i} \in A, g_{i} \in G$. Moreover, any element $g \in G^{*}$ admits a unique decomposition of the following type, which is called a canonical form of $g$ :

$$
g=h g_{1} v^{s_{1}} \ldots g_{n} v^{s_{n}} g_{n+1}
$$

where $h \in v^{C}, g_{i} \in \mathcal{R}, s_{i} \in S, s_{i} \neq 0$ and $g_{i} \neq 1$ (except, maybe, $g_{1}, g_{n+1}$ ). We suppose here that $g$ itself is a canonical form for $g \in G$.

Any product of type (11) (or, more precisely, any sequence $\left(g_{1}, v_{1}^{a_{1}}, g_{2}, \ldots, v^{a_{n}}, g_{n+1}\right)$ ) can be reduced to a canonical form by the following rewriting process:

- if $a_{n} \in C$, then

$$
g_{1} v_{1}^{a_{1}} \cdots g_{n} v^{a_{n}} g_{n+1} \rightarrow g_{1} v^{a_{1}} \cdots g_{n-1} v^{a_{n-1}}\left(g_{n} v^{a_{n}} g_{n+1}\right),
$$

so we get a shorter decomposition of $g$ and can apply induction;

- if $a_{n} \notin C$, then

$$
g_{1} v^{a_{1}} \cdots g_{n} v^{a_{n}} g_{n+1} \longrightarrow g_{1} v^{a_{1}} \cdots\left(g_{n} v^{k+t}\right) v^{s_{n}} \bar{g}_{n+1},
$$

where $a_{n}=k+s_{n}, g_{n+1}=v^{t} \bar{g}_{n+1}, k, t \in C, s_{n} \in S$; 
- if $\left(g_{n} v^{k+t}\right) \in C$, then

$$
g_{1} v^{a_{1}} \ldots v^{a_{n-1}}\left(g_{n} v^{k+t}\right) v^{s_{n}} \bar{g}_{n+1} \longrightarrow g_{1} v^{a_{1}} \ldots\left(g_{n-1} g_{n} v^{k+t}\right) v^{a_{n-1}+s_{n}} \bar{g}_{n+1},
$$

and we get a shorter decomposition of $g$. After that the process is performed by induction.

- if $g_{n} v^{k+t} \notin C$, then one can apply the rewriting process to $g_{1} v^{a_{1}} \cdots v^{a_{n-1}} g_{n} v^{k+t}$, keeping the tail unchanged.

It is easy to see that these transformations carry $g$ to the canonical form.

It is more convenient to use the reduced form of elements of $G^{*}$. By definition, an element $g$ is in reduced form

$$
g=g_{1} v^{a_{1}} \ldots g_{n} v^{a_{n}} g_{n+1}
$$

if $a_{i} \notin C, i=1, \ldots, n, g_{j} \notin C, j=2, \ldots, n$.

From the rewriting process it is clear that a form of type (1) is reduced iff the number $n$ is minimal through all decompositions of $g$ of type (1).

Propostion 6 Let

$$
\begin{gathered}
g=u_{1} v^{a_{1}} \ldots u_{m} v^{a_{m}} u_{m+1} \\
g=w_{1} v^{b_{1}} \ldots w_{n} v^{b_{n}} w_{n+1}
\end{gathered}
$$

be two reduced forms of an element $g$. Then:

1. $m=n$ and $a_{1}=b_{1}+k_{1}, \ldots, a_{m}=b_{m}+k_{m}$ for some $k_{i} \in C$;

2. $u_{m+1}=v^{t_{m}} w_{m+1}, u_{m}=v^{t_{m-1}} w_{m} v^{-t_{m}-k_{m}}, \ldots, u_{1}=w_{1} v^{-t_{1}-k_{1}}$ for some $t_{j} \in C, j=1, \ldots, m$.

By the rewriting process.

For a direct extension of a centralizer $C$ by a group $H$ (i.e. when $A=$ $C \oplus H)$, the group $H$ is itself a system of representatives of $A$ by $C(H=S)$. In this case it is very convenient to use semicanonical forms of elements.

By definition, a reduced form (21)

$$
g=g_{1} v^{s_{1}} \cdots v^{s_{n}} g_{n+1}
$$

is semicanonical, iff $1 \neq s_{i} \in S$ for all $i$.

From proposition 6 one can easily deduce the following 
Corollary 1 Any two semicanonical forms of $g$ can be transformed from one to another by a finite sequence of commutations of the form $v^{s} v^{t}=v^{t} v^{s}$, where $t \in C, s \in S$.

Suppose now that the notions of canonical, semicanonical and reduced forms of elements of the group $G$ are already introduced. In this case one can extend them to the group $G^{*}$.

We will say that canonical (semicanonical, reduced) forms of an element

$$
g=h g_{1} v^{a_{1}} \cdots v^{a_{n}} g_{n+1}
$$

agree with those on $G$, iff the elements $h, g_{1}, \ldots, g_{n+1}, v$ are in canonical (semicanonical, reduced) forms in the group $G$.

Now we are able to introduce the corresponding forms of elements of tree and iterated tree extensions of centralizers.

Let $G^{*}$ be the union of a chain of groups

$$
G=G_{0} \hookrightarrow G_{1} \hookrightarrow \cdots \hookrightarrow G_{\alpha} \hookrightarrow \cdots
$$

where every $G_{\alpha+1}$ is obtained from $G_{\alpha}$ by extension of centralizers, and $G_{\gamma}=$ $\lim _{\rightarrow} G_{\alpha}$ for a limit ordinal $\gamma$. Then the notions of canonical (semicanonical, reduced) forms are introduced on $G_{\alpha+1}$ agreeably with those on $G_{\alpha}$. For a limit ordinal $\gamma$ these forms are inherited form all the previous terms of the chain. Then canonical (semicanonical, reduced) forms on the group $G^{*}$ are well-defined.

\section{Groups with conjugately separated maxi- mal abelian subgroups}

In this section we introduce the class of $\mathrm{CSA}^{*}$-groups which will play a fundamental role in our investigation of $A$-free groups, tensor completions and groups with length functions. This class is quite wide; for example, it contains all torsion-free hyperbolic groups, all groups acting freely on $\Lambda$-trees, and all universally free groups.

Definition 10 A subgroup $H$ of a group $G$ is called conjugately separated (or malnormal) if $H \cap H^{x}=1$ for any $x \in G \backslash H$. 
Definition 11 A group $G$ is called a CSA-group if all its maximal abelian subgroups are conjugately separated.

It is clear that every abelian group is a CSA-group.

The following proposition gives us some intuition about CSA-groups.

Propostion 7 Let $G$ be a CSA-group. Then the following statements are true:

1. (SA-property) if $M_{1}$ and $M_{2}$ are distinct maximal abelian subgroups of $G$, then $M_{1} \cap M_{2}=1$;

2. any maximal abelian subgroup of $G$ coincides with its normalizer.

1) If $1 \neq x \in M_{1} \cap M_{2}$ and $y \in M_{2} \backslash M_{1}$, then $x \in M_{1}^{y} \cap M_{1}$, so $M_{1}^{y} \cap M_{1} \neq 1$.

2) is evident.

Definition 12 A group $G$ is called commutative transitive if the relation " $a$ commutes with $b$ " is transitive on the set $G \backslash 1$;

Remark 4 The following conditions are equivalent to one another:

1. a group $G$ has $S A$-property;

2. a group $G$ is commutative transitive;

3. in a group $G$ the centralizer of any nontrivial element is abelian.

Remark 5 Proposition 7 shows that the class CSA is a subclass of the class of groups with transitive commutation. The following example proves that CSA is a proper subclass of these classes.

Let $G$ be a free metabelian group. Then all proper centralizers of $G$ are commutative. But $G$ has a normal abelian subgroup, so it is not a CSAgroup.

In fact, the transitivity of commutation is a very strong property and implies some other interesting features. We collect them in the following 
Propostion 8 Let $G$ be a group such that commutation is an equivalence relation on $G \backslash\{1\}$. Then

1. the centralizer of any nontrivial element of $G$ is a maximal abelian subgroup of $G$; conversely, any maximal abelian subgroup of $G \neq 1$ is a centralizer of some nontrivial element;

2. if $G$ is torsion-free, then for $x, y \in G x^{n}=y^{m} \Longrightarrow[x, y]=1$;

$x^{n}=y^{n} \Longrightarrow x=y$;

3. if $G$ is nonabelian, then its center is trivial;

4. if $G$ is nonabelian, then it is indecomposable into a nontrivial direct product.

1), 3) and 4) are quite evident. The second implication in 2) is a corollary of the first one. Let us prove the first claim of 2).

Suppose $x^{n}=y^{m}$. Then the neighboring terms in the sequence $x, x^{m}, y^{n}, y$ are nontrivial and commute pairwise; by transitivity of commutation we obtain $[x, y]=1$.

Remark $6 G$ is a CSA-group iff all its centralizers of nontrivial elements are abelian and conjugately separated (see statement 1 of proposition 8).

Thus, CSA is a property of centralizers. Let us consider a typical situation of conjugate unseparability. A cyclic subgroup $\langle y\rangle=M<G$ is not conjugately separated iff there is some $x \in G$ such that $M^{x} \cap M \neq 1$, or, equivalently, $x$ and $y$ satisfy the identity $x^{-1} y^{r} x=y^{s}$ for some integers $r$ and $s$. One-relator groups with this relation have a special name and play a crucial role in what follows. Groups of the form

$$
G_{r, s}=\left\langle a, b \mid a^{-1} b^{r} a=b^{s}\right\rangle, r, s \in \mathbf{Z}, r>0,
$$

are called Baumslag - Solitar groups [0]. We will need the following properties of these groups:

- if $r=s=1$, then $G_{r, s} \simeq \mathbf{Z} \times \mathbf{Z}$ is an abelian group; 
- if $r=1$ and $s \neq 1$, then $G_{r, s}$ is nonabelian solvable and for $s \neq-1$

$$
G_{1, s}=\left\langle a, b \mid a^{-1} b a=b^{s}\right\rangle \simeq \mathbf{Z}\left[\frac{1}{s}\right] \succ \mathbf{Z}
$$

where $\mathbf{Z} \simeq\langle a\rangle, \mathbf{Z}\left[\frac{1}{s}\right] \simeq \operatorname{n.cl}(b)$ - the normal closure of $\langle b\rangle$;

for $s=-1$

$$
G_{1, s}=\left\langle a, b \mid a^{-1} b a=b^{-1}\right\rangle \simeq\langle b\rangle \rtimes\langle a\rangle .
$$

- if $r>1,|s|>1$, then $G_{r, s}$ is a nonsolvable group and $\left[a^{-1} b a, b\right] \neq 1$ (see, for example, [10, p.281]). The case $r>1, s= \pm 1$ coincides with the second one after renaming the generators.

In the following proposition we collect some properties of CSA-groups.

Propostion 9 Let $G$ be a nonabelian CSA-group. Then

1. centralizers of nontrivial elements of $G$ are abelian and coincide with maximal abelian subgroups of $G$ (in particular, commutation is transitive);

2. G has no nontrivial normal abelian subgroups;

3. G has a trivial center;

4. $G$ is directly indecomposable;

5. G has no non-abelian solvable subgroups;

6. G has no nonabelian Baumslag - Solitar subgroups;

7. if $G$ is torsion-free, then $G$ is a $D$-group, i.e. the operations of extracting roots in $G$ are defined uniquely.

1) is follows from item 3) of proposition 7 and item 1) of proposition 8. 2 ) is clear, and 3) is a corollary of 2). 4) follows from 1) and item 4) of proposition 8. In proposition 10 below it will be proved that a subgroup of a CSA-group is again a CSA-group. So to prove 5) one only needs to prove that a non-abelian solvable group is not a CSA-group. But the last statement follows from 2). To prove 6), it suffices to prove that a non-abelian Baumslag-Solitar group $G_{r, s}$ is not a CSA-group. If $r=1$ and $s \neq 1$, then 
$G_{r, s}$ is nonabelian solvable (see remarks before the proposition), and hence by 5 ) it is not a CSA-group. If $r>1$ and $|s|>1$, then $\left[a^{-1} b a, b\right] \neq 1$. Suppose now that $G_{r, s}$ is a CSA-group, then commutation on nontrivial elements of $G$ must be transitive. In the sequence $a^{-1} b a, a^{-1} b^{r} a, b^{s}, b$ all neighbours commute, but the first and the last elements do not - a contradiction with the transitivity of commutation. 7) follows from item 3) of proposition 7 and item 2) of proposition 8 .

Propostion 10 1. The class of groups CSA is finitely universally axiomatizible.

2. The class CSA is closed under ultraproducts.

3. The class CSA is closed under taking subgroups.

To prove 1) it suffices to write down the conditions of commutativity of centralizers and their conjugate separation by universal formulas. This is straightforward. 2) follows from the fact that this class is axiomatizible. 3) follows from the universal axiomatization of this class.

Free groups are principal examples of nonabelian CSA-groups. The following results show that the class CSA contains almost all groups which are "close" to free groups.

Propostion 11 Let $G$ be a torsion-free group such that the centralizer of any nontrivial element is cyclic. Then $G$ is a CSA-group.

Let $G$ satisfy conditions of Proposition 11. Then proper centralizers of $G$ are abelian (in particular, commutation is transitive on $G \backslash\{1\}$ ), and by remark 4 we only need to prove that these centralizers are conjugately separated. Consider any maximal abelian subgroup $M$ of $G ; M=\langle y\rangle$. Suppose that for some $x \in G \backslash M$ we have $M \cap M^{x} \neq 1$. This means that $x^{-1} y^{s} x=y^{r}$ for some integers $s, r$. Let us denote $z=x^{-1} y x$. Then $z^{s}=y^{r}$ and by proposition $8[z, y]=1$. So $z \in\langle y\rangle$ and $x^{-1} y x=y^{p}$ for some integer $p$. Therefore the subgroup $\langle x, y\rangle$ is a quotient group of the Baumslag - Solitar group $G_{1, p} \simeq\left\langle a, b \mid a^{-1} b a=b^{p}\right\rangle$ (see remarks before proposition 9) under the epimorphism $\phi: a \mapsto x, b \mapsto y$. We know that $G_{1, p}=\operatorname{n.cl}\langle b\rangle>\triangleleft\langle a\rangle$, where n.cl $\langle b\rangle \simeq \mathbf{Z}\left[\frac{1}{p}\right],\langle a\rangle \simeq \mathbf{Z}$. Suppose that $b_{1} a^{n} \in \operatorname{ker} \phi$, i.e. $y_{1} x^{n}=1, y_{1} \in$ 
n.cl $(y)$. Then in the sequence $y, y_{1}, x^{n}, x$ neighbours commute, and hence by transitivity of commutation $[y, x]=1$ (but this contradicts to our choice of $x$ and $y$ ) or else $y_{1}$ or $x^{n}$ is trivial. The last case implies that $y_{1}=1, n=0$. Hence $\langle x, y\rangle \simeq G_{1, p}$ - a contradiction, because $\mathbf{Z}\left[\frac{1}{p}\right]$ is a subgroup of $G_{1, p}$ and is not cyclic.

Propostion 12 1. Any torsion-free hyperbolic group is a CSA-group.

2. Any group acting freely on a $\Lambda$-tree is a CSA-group.

3. Any universally free group is a CSA-group.

$\square$ It is known (see, for example, [6]) that the centralizer of any element of infinite order in a hyperbolic group $G$ has a cyclic subgroup of finite index (i.e. the stabilizer is virtually cyclic). So we only need to prove that any torsion-free virtually cyclic group $H$ is infinite cyclic. It is clear that $H$ is finitely generated and all subgroups of $H$ are also virtually cyclic. By induction on the number of generators of $H$, we may assume that $H$ is 2generated, $H=\langle a, b\rangle$ and moreover $\langle b\rangle \triangleleft H, a^{m} \in\langle b\rangle, a^{-1} b a=b^{n}$, for some $n, m$. This implies $b=a^{-m} b a^{m}=b^{n^{m}}$. Therefore $1=n^{m}$. Hence $n= \pm 1$ and $[a, b]=1$ or $\left[a^{2}, b\right]=1$. In the first case $H$ is abelian torsion-free and virtually cyclic, therefore $H$ is a cyclic group. In the second case $a^{2}$ is a central element of $H$, and so is the element $a^{2 m}=b^{k}$ for some integer $k$. But $a^{-1} b^{k} a=b^{-k}$, and hence $b^{k}=b^{-k}$, which contradicts to the torsion-free condition on $G$.

Statement 2) is proved in [3, corollary 1.9].

In [17] it was proved that every universally free group acting freely on some $\Lambda$-tree. Now 3) follows from 2).

\section{Preservation of the CSA-property under free products, direct limits, and abelian ex- tensions of centralizers}

In this section we will prove that the CSA-property is preserved under free products and abelian extensions (tree extensions or iterated tree extensions) 
of centralizers for groups without elements of order 2 (non-abelian CSA groups automatically do not contain elements of order 2). In any case it is preserved under direct limits.

Definition 13 The subclass CSA* of CSA consists of all groups without elements of order 2.

Remark 7 Any non-abelian CSA-group is also CSA*-group.

If $G$ is non-abelian CSA-group and $u^{2}=1$ for some nontrivial $u \in G$, then the normal closure of $u$ in $G$ is also non-abelian (otherwise it would contradict to CSA-property). Hence, there are two noncommutative involutions in $G$, they generate non-abelian metabelian subgroup of $G$ - contradiction with CSA-property.

Theorem 4 The class $C S A^{*}$ is closed under free products.

Let $G_{1}$ and $G_{2}$ be CSA*-groups and $G=G_{1} * G_{2}$. First of all, $G$ has no elements of order two if $G_{1}$ and $G_{2}$ do not (see [10, cor. 4.4.5]). By the Kurosh Subgroup Theorem a maximal abelian subgroup $M$ of the group $G$ has one of the following two types:

a) $M \leq G_{i}^{g}$ for some $g \in G, i=1,2$.

b) $M=\langle z\rangle$ is an infinite cyclic group generated by an element $z$ of cyclically reduced length $\|z\| \geq 2$.

By definition, $\|z\|=\min \{|y| \mid y$ is cyclically reduced and conjugate to $z\}$. Clearly, $\|z\| \geq 2$ iff $z \notin G_{i}^{g}$ for any $g \in G, i=1,2$. We claim that all centralizers of nontrivial elements in $G$ are abelian and, consequently, commutation in $G \backslash\{1\}$ is transitive. Indeed, let $1 \neq a \in G, b, c \in C_{G}(a)$. There are maximal abelian subgroups $M, N<G$ with $a, b \in M$ and $a, c \in N$. We have the following cases:

1. $M<G_{i}^{x}, N<G_{i}^{y}, i=1,2, x, y \in G$. Then $G_{i} \cap G_{i}^{x y^{-1}} \neq 1$, hence $x y^{-1} \in G_{i}$, and $G_{i}^{x}=G_{i}^{y}$. So $a, b, c \in G_{i}^{x}$. But since $G_{i}$ is a CSA-group, it follows that $[b, c]=1$. 
2. The case $M<G_{i}^{x}, N<G_{j}^{y}, i \neq j$ is impossible.

3. The case $M=\langle z\rangle$ with $\|z\| \geq 2, N<G_{i}^{x}$ is impossible, because $a \in M$ implies $\|a\| \geq 2$, and on the other hand, $a \in N$ implies $\|a\|=1$.

4. $M=\langle z\rangle, N=\langle y\rangle,\|z\| \geq 2,\|y\| \geq 2$. Then $a=z^{n}=y^{l}$. So $y^{l} \in Z(H)$, where $H=\langle z, y\rangle$. By the Kurosh subgroup theorem, $H$ is a free product $H=F *\left(* G_{i}^{\alpha}\right)$, where $F$ is a free group. Since $H$ has a nontrivial centre, it equals to one of the factors. If $H=F$, then $H$ is cyclic, and $[z, y]=1$. Therefore $[b, c]=1$. The case $H<G_{i}^{\alpha}$ is impossible because $\|z\| \geq 2$.

This proves that $C_{G}(a)$ is abelian.

Suppose that $M \leq G_{i}^{g}$, then $M$ is conjugately separated iff its automorphic image $M^{g^{-1}}$ is conjugately separated. Conjugating by $g^{-1}$ if necessary, we may assume that $M \leq G_{i}$. It is a well-known property of free products (see [10, cor. 4.1.5]) that if $x$ is not in $G_{i}$, then $G_{i}^{x} \cap G_{i}=1$. Hence if $M^{x} \cap M \neq 1$, then $x \in G_{i}$, but $G_{i}$ is a CSA-group, therefore $x \in M$. Now suppose that $M=\langle z\rangle$ and $\|z\| \geq 2$. If $M^{x} \cap M \neq 1$, then $x^{-1} z^{n} x=z^{m}$ for some $n$ and $m$. Hence $\left\|z^{n}\right\|=\left\|z^{m}\right\|$. But it is easy to see that $\left\|z^{n}\right\|=|n| \cdot\|z\|$, and the equality above implies that $|m|=|n|$. In the case $n=m$ we have $\left[x, z^{n}\right]=1$, and by transitivity of commutation $[x, z]=1$. Consequently, $x \in\langle z\rangle$. Assume now that $n=-m$. In this case $x^{-1} z^{n} x=z^{-n}$, which implies $x^{-2} z^{n} x^{2}=z^{n}$. So $\left[x^{2}, z^{n}\right]=1$, and by transitivity $[x, z]=1$, as above.

The following theorem plays the main part in the rest of the paper.

Theorem 5 The class CSA* is closed under extensions of centralizers by abelian groups without elements of order 2.

Let $G$ be a $\mathrm{CSA}^{*}$-group, $C=C_{G}(v)$ the centralizer of an element $v \in G$, and $\phi: C \hookrightarrow A$ an embedding of $C$ into an abelian group $A$ which has no elements of order two. We need to prove that the extension $G(v, A)$ is also a CSA*-group. The group $G(v, A)$ has no elements of order two (匹10, cor. 4.4.5]). Therefore it remains to check the CSA-property. Before proving this, we establish a lemma which describes centralizers in the group $G(v, A)$.

Lemma 1 Let $x \in G(v, A)$. Then: 
1. if $x \in G^{g}$, then $C(x) \leq G^{g}$;

2. if $x \in A^{g}$, then $C(x)=A^{g}$;

3. if $\|x\| \geq 2$, then $C(x)=\langle z\rangle$ and $\|z\| \geq 2$.

We will use the following description of commuting elements in free products with amalgamation (see [10]): $[x, y]=1$ iff one of the following conditions holds:

- $x$ or $y$ belongs to some conjugate of the amalgamated subgroup $C=$ $C_{G}(v)$;

- neither $x$ nor $y$ is in a conjugate of $C$, but $x$ is in a conjugate of a factor ( $G$ or $A$ ), then $y$ is in the same conjugate of the factor;

- neither $x$ nor $y$ is in a conjugate of a factor, then $x=g^{-1} c g z^{n}, y=$ $g^{-1} c^{*} g z^{m}$, where $c, c^{*} \in C$, and $g^{-1} c g, g^{-1} c^{*} g$ and $z$ commute pairwise.

Let us consider these three possible cases.

Suppose that $x \in C^{g}, y \in G(v, A)$, and $[x, y]=1$. We can assume (conjugating by $g^{-1}$ if necessary) that $x \in C$. Let $y=c g_{1} a_{1} \ldots g_{n} a_{n}$ be the canonical form of $y$, where $c \in C, g_{i}$ are right representatives of $G \bmod C$, $a_{i}$ are right representatives of $A \bmod C$, and $g_{n} \neq 1$. Then $x y=y x$ implies

$$
x c g_{1} a_{1} \ldots g_{n} a_{n}=c g_{1} a_{1} \ldots g_{n} a_{n} x=c g_{1} a_{1} \ldots g_{n} x a_{n}
$$

Hence $g_{n} x=x_{1} g_{n}$ for some $x_{1} \in C$ or $g_{n} x g_{n}^{-1}=x_{1}$. But $G$ is a CSA-group and $C$ is its nontrivial centralizer - a contradiction. Therefore if $[x, y]=1$, then $y=c a_{n} \in A$. And we have case 2) of the lemma.

Suppose that $x$ belongs to a conjugate of a factor $G$ or $A$, but not to $C$. Then $y$ is in the same factor, and we have case 1) or 2) of the lemma.

Finally, suppose that $\|x\| \geq 2$ and $[x, y]=1$ for $y \in G(v, A)$. Then $x=g^{-1} c g z^{n}, y=g^{-1} c^{*} g z^{m},\|z\| \geq 2$, and $z, g^{-1} c g$ and $g^{-1} c^{*} g$ commute in pairs. Conjugating by $g^{-1}$, we reduce the problem to the case $x=c z_{1}^{n}, y=$ $c^{*} z_{1}^{m}, z_{1}=g z g^{-1}$. If $c \neq 1$ or $c^{*} \neq 1$, then $z_{1} \in A$ according to the proof above, but this contradicts the condition $\|z\| \geq 2$. So $c=1, c^{*}=1$ and we have the case 3 ) of the lemma. 
Corollary 2 1. All centralizers of nontrivial elements of the group $G(v, A)$ are abelian, and the group $G(v, A)$ is commutative transitive;

2. The cenralizer of the element $v$ in the group $G(v, A)$ is exactly the subgroup $A$.

Corollary 3 A maximal abelian subgroup of $G(v, A)$ can be of one of the following three types:

1. $M^{g}$, where $M$ is a maximal abelian subgroup of $G, M \neq C^{f}$ ( $M$ is not conjugate to $C$ ) and $g \in G(v, A)$;

2. $A^{g}, g \in G(v, A)$;

3. $\langle z\rangle$, where $\|z\| \geq 2$.

The following lemma describes conjugated elements in the group $G(v, A)$.

Lemma 2 Let $g$ be a cyclically reduced element of $G(v, A)$. Then:

a) if $g$ is conjugate to an element $h$ in some factor, then $g$ and $h$ are in the same factor and are conjugate in it;

b) if $g$ is conjugate to a cyclically reduced element $p_{1} \cdots p_{n}$, where $n \geq$ 2 , then $g$ can be obtained by cyclically permuting $p_{1}, \ldots p_{n}$ and then conjugating by an element from $C$.

There is a description of conjugated elements in arbitrary free products with amalgamation [10]. In a general situation, one has our cases a), b) and one more. Namely, $g$ is conjugate to some $c \in C$. In this case, according to the description in [10], there are elements $c_{i} \in C$ such that in the sequence $c, c_{1}, \ldots, c_{n}, g$ consecutive terms are conjugate in a factor. In our case the factor $A$ is abelian, therefore $g$ and $c$ are conjugate in $G$.

Proof of the theorem. Let $N=M^{g}$ be a maximal abelian subgroup of $G$ of the type 1). We need to prove that $N^{x} \cap N \neq 1 \Longrightarrow x \in N$ for any $x \in$ $G(v, A)$. Conjugating if necessary, we can assume that $N=M \leq G$. Suppose that there exist nontrivial elements $f, h \in M$ such that $f^{x}=h$. According to statement a) above, $f^{y}=h$ for some $y \in G$. But then $M \cap M^{y} \neq 1$, and 
consequently $y \in M$ and $f=h$ (because $G$ is a CSA-group). This implies that $[f, x]=1$ and $x \in G$. Hence $x \in M$.

Now let $N=A^{g}, g \in G(v, A)$. Again, we can consider only the case $N=A$. Suppose that $A \cap A^{x} \neq 1$ for some $x \in G(v, A)$. Then $f^{x}=h$ for some $f, h \in A$. If $f$ or $h$ is not in $C=C_{G}(v)$, then by lemma 2 a) $f$ and $h$ are conjugate in $A$, but $A$ is abelian, so $f=h$. If both $g$ and $h$ are in $C$, then they are both in $A$ and $G$, and we can apply a) as before.

Let $N=\langle z\rangle$ be an infinite cyclic group, $\|z\| \geq 2$. Suppose that $N \cap N^{x} \neq$ 1. Then $\left(z^{n}\right)^{x}=z^{m}$ for some integers $m$ and $n$. Now we are in the case b) of the description. To finish the proof in this case, one can repeat word by word the proof from the last part of theorem 4 .

Theorem 6 The class CSA is closed under direct limits.

Let $\mathcal{G}=\left\{G_{i}, \phi_{j}^{i}, I\right\}$ be an inductive system of CSA-groups $G_{i}, i \in I$, and $G^{*}=\lim \longrightarrow G_{i}$. The following lemma describes centralizers of nontrivial elements in $G^{*}$.

Lemma 3 Let $G^{*}=\lim \longrightarrow G_{i}$ be a direct limit of $C S A$-groups $G_{i}, i \in I$. If $M$ is a maximal abelian subgroup of $G^{*}$, then for any $i \in I M_{i}=G_{i} \cap M$ is either maximal abelian in $G_{i}$ or trivial. Moreover, $M=\lim \longrightarrow M_{i}$

First of all, commutation is transitive on $G^{*} \backslash\{1\}$. Indeed, any three elements $x, y, z \in G^{*}$ are in some $G_{i}$, but $G_{i}$ is a CSA-group.

Let $M_{i}$ be nontrivial. If $M_{i}$ is not maximal in $G_{i}$, then $\left[M_{i}, x\right]=1$ for some $x \notin M_{i}$. Let us prove that $x \in M$. By transitivity of commutation, $M$ is a centralizer of some element $z \in G$. There exists $k$ such that $i<k$ and $x, z \in G_{k}$. From transitivity of commutation we deduce that $[x, z]=1$, and therefore $x \in M$, which contradicts to the condition $x \notin M_{i}$. The equality $M=\lim _{\longrightarrow} M_{i}$ is evident.

Let us continue the proof of theorem 6 .

Suppose that $M$ is a maximal abelian subgroup of $G^{*}$, and for some $f \in G^{*} M \cap M^{f} \neq 1$. Then $x=y^{f}$ for some $x, y \in M$. We can choose $k \in I$ such that $x, y, f \in G_{k}$ and therefore $M_{k} \cap M_{k}^{f} \neq 1$ in $G_{k}$. Hence $f \in M_{k}<M$. This proves that $G^{*}$ is a CSA-group.

Theorem 7 The class $C S A^{*}$ is closed under tree abelian extensions of cenralizers and iterated tree abelian extensions of centralizers by abelian groups without elements of order 2. 
$\square$ By definition, a tree (iterated tree) extensions of centralizers of a group $G$ is constructed by transfinite induction on ordinals: $G=G_{0}<G_{1}<\cdots<$ $G_{\alpha}<\cdots$. Following this construction, it suffices to prove by induction that every term of this chain lies in the class $\mathrm{CSA}^{*}$ if the initial group $G$ belongs to CSA*. For a nonlimit ordinal, this was proved in theorem 5 , for a limit ordinal in theorem 6 .

\section{Tensor completion of CSA-groups}

In [15], using general category theory, we proved that for any group $G$ and any ring $A$ the tensor completion $G^{A}$ exists, but we did not point out any idea of what this construction is explicitly.

In this section we, following the method of G. Baumslag [ [1], describe the explicit construction of a tensor completion of an arbitrary CSA ${ }^{*}$-group $G$ using extensions of centralizers. In fact, $G^{A}$ appears as an iterated tree extension of centralizers in $G$ for a very wide class of rings $A$. In particular, $G^{A}$ is again a $\mathrm{CSA}^{*}$-group, and we obtain a lot of information about it. Moreover, we can apply the technique of amalgameted free products to investigate $G^{A}$. Thus, one has the canonical and reduced forms of elements in

$G^{A}$, descriptions of commuting and conjugating elements, elements of finite order and so on.

We will construct the $A$-completion of a group $G$ step by step, on each stage obtaining partial $A$-groups.

\section{Abelian case}

In the abelian case the construction of tensor completion is very similar to ordinary tensor multiplication by the ring $A$ (one only needs to make the existing partial action of $A$ on $M$ agree with the action of $A$ on $M \otimes A$ ).

Let $M$ be a partial right $A$-module (i.e. a partial abelian $A$-group). Let us consider the ordinary tenzor product $M \otimes_{\mathbf{z}} A$ of abelian group $M$ by the ring $A$ over $\mathbf{Z}$. Then the tensor $A$-completion of the partial $A$-module $M$ can be obtained by factorizing $M \otimes_{\mathbf{z}} A$ by all relations of the type $(x \alpha, \beta)-$ $(x, \beta) \alpha, x \in M, \alpha, \beta \in A$.

The following proposition is a copy of the corresponding result from the theory of modules. 
Propostion 13 1. Let $M$ be a torsion-free abelian group, and $A$ a ring with a torsion-free additive group. Then $i: M \longrightarrow M \otimes A$ is injective, and $M \otimes A$ is torsion-free.

2. Let $M$ be a partial $A$-module without elements of order 2, and $A$ a ring whose additive group has no elements of order 2. Then $M \otimes A$ has no elements of order 2.

\section{Non-abelian case}

Before getting into details for the noncommutative case, we will briefly describe the plan of an attack. By the definition of an $A$-group, the centralizers of $G^{A}$ must be $A$-modules. This gives us a hint. The main idea is to extend all centralizers of $G$ up to $A$-modules, adding a minimal number of new relations. Centralizers in a CSA-group are abelian, and we already know their $A$-completions. But we need to have this tensor completion of given maximal abelian subgroups $M$ of $G$ agree with all the other elements. The best way to do that is to extend the centralizers of $G$ by the $A$-module $M \otimes A$ under the canonical monomorphism $i: M \longrightarrow M \otimes A$. Then, using conjugate separation of the subgroup $M$, we can extend the action of $A$ on $M \otimes A$ to all conjugates of $M$ (in order to satisfy axiom 3 of an $A$-group, according to which the action of $A$ on $G$ commutes with all conjugations). To make this plan work, we need to put some natural restrictions on $G$ and $A$, namely, to assume that the homomorphism $i: M \longrightarrow M \otimes A$ is injective, and $A^{+}$contains no elements of order 2 (i.e. $A^{+}$is a $\mathrm{CSA}^{*}$-group).

Before proving the main theorem, let us describe the construction of complete tensor extension of centralizers of $G$ by the ring $A$. We will assume that $G$ is a non-abelian CSA-group and all maximal abelian subgroups of $G$ are faithful over the ring $A$.

Let $\mathcal{C}=\left\{C_{i}, i \in I\right\}$ be the set of all centralizers of $G$ which satisfies the following conditions $(\mathrm{S})$ :

- any $C \in \mathcal{C}$ is not an $A$-module (as a partial abelian $A$-subgroup in $G$ );

- any two centralizers from $\mathcal{C}$ are not conjugate in $G$;

- any centralizer in $G$ which is not an $A$-module is conjugate to an element of $\mathcal{C}$. 
Then we can form the set $\mathcal{H}_{A}=\left\{C_{i} \otimes A \mid i \in I\right\}$ and the set of canonical embeddings $\Phi_{A}=\left\{\phi_{i}: C_{i} \hookrightarrow C_{i} \otimes A \mid i \in I\right\}$. Consider the tree extension of centralizers of this special type:

$$
G^{*}=G\left(\mathcal{C}, \mathcal{H}_{A}, \Phi_{A}\right)=G(\mathcal{C}, A)
$$

Now we can iterate this construction up to level $\omega$ :

$$
G=G^{(0)}<G^{(1)}<G^{(2)}<\cdots<G^{(n)}<\cdots
$$

where $G^{(n+1)}=G^{(n)}\left(\mathcal{C}_{n}, A\right)$, and the set $\mathcal{C}_{n}$ of centralizers in the group $G^{(n)}$ satisfies the condition $(\mathrm{S})$.

Definition 14 The union $\bigcup_{n \in \omega} G^{(n)}$ of the chain (3) is called complete tensor extension of centralizers of $G$ by the ring $A$.

Theorem 8 Let $A \geq \mathbf{Z}$ be a ring without additive elements of order 2 , and let a nonabelian partial $A$-group $G$ be a $C S A$-group. If all maximal abelian subgroups of $G$ are faithful over $A$, then the tensor completion of $G$ by $A$ is the complete tensor extension of centralizers of $G$ by the ring $A$.

Let us prove by induction on $n$ that each group $G^{(n)}$ from the chain (3) meets the conditions of the theorem and satisfies some special universal property. So now we will focus our attention on the tree extension of centralizers $G^{*}=G(\mathcal{C}, A)$, which was described above.

By the construction of a tree extension (see section 2 ), $G^{*}$ is the union of the chain of groups

$$
G=G_{0} \hookrightarrow G_{1} \hookrightarrow \cdots \hookrightarrow G_{\alpha} \hookrightarrow \cdots \hookrightarrow G_{\delta}=G^{*}
$$

where the set of centralizers $\mathcal{C}=\left\{C_{\alpha} \mid \alpha<\delta\right\}$ is well-ordered, $C_{\alpha}=C_{G}\left(v_{\alpha}\right)$, and by definition:

$$
\begin{aligned}
& G_{\alpha+1}=G_{\alpha}\left(v_{\alpha}, C_{\alpha} \otimes A\right) ; \\
& G_{\gamma}=\bigcup_{\alpha<\gamma} G_{\alpha} \text { for a limit ordinal } \gamma .
\end{aligned}
$$

Lemma 4 For any $1 \neq g \in G$ the centralizer $C_{G^{*}}(g)$ of $g$ in $G^{*}$ is isomorphic to $C_{G}(g) \otimes A$. 
By the choice of the set $\mathcal{C}$ the centralizer $C_{G}(g)$ is conjugate in $G$ to some centralizer $C_{\alpha}=C_{G}\left(v_{\alpha}\right) \in \mathcal{C}$, or $C_{G}(g)$ is an $A$-module in $G$. In the first case, the centralizer $C_{G}\left(v_{\alpha}\right)$ has to be extended exactly once on the level $\alpha$, and by corollary 2 of lemma 1 , the extended centralizer is equal to $C_{\alpha} \otimes A=C_{G^{*}}\left(v_{\alpha}\right)$. Therefore the centralizer $C_{G^{*}}(g)$ in $G^{*}$ is conjugate to $C_{G^{*}}\left(v_{\alpha}\right)$, and hence $C_{G^{*}}(g) \simeq C_{G}(g) \otimes A$. In the second case $C_{G}(g)$ is an $A$-module, so $C_{G^{*}}(g) \simeq C_{G}(g) \otimes A$.

Lemma $5 G^{*}$ is a partial $A$-group, and the action of $A$ on $G$ is defined in $G^{*}$.

By lemma 4, in the group $G^{*}$ the centralizer $M=C_{G^{*}}(g)$ of any nontrivial element $g \in G$ is isomorphic to the group $C_{G}(g) \otimes A$ which has a canonial structure of an $A$-module. Using this isomorphism, the action of $A$ on $C_{G}(g) \otimes A$ can be induced on $M$. By theorem $7, G^{*}$ is a CSAgroup, hence $M$ is conjugately separated, and we can extend this action correctly to all conjugates of $M$ in $G^{*}$ to satisfy axiom 3) from the definition of an $A$-group. All maximal abelian subgroups in $G^{*}$ are separated, so the intersections $M_{1} \cap M_{2}$ are trivial, and we have a well-defined action of $A$ on the group $G$ and all its conjugates. Moreover, axioms 1)-4) from the definition of an $A$-group hold in $G^{*}$.

Lemma $6 G^{*}$ is a CSA-group, and all its maximal abelian subgroups are faithful over $A$.

From the general theory of free products with amalgamation (see [10]) we know that elements of finite order lie in conjugates of factors. By proposition 13, the tensor completion $M \otimes A$ of any maximal abelian subgroup $M$ of $G$ has no elements of order 2. Therefore by transfinite induction on the construction of the tree extension $G^{*}$ we conclude that $G^{*}$ has no elements of order 2. $G^{*}$ is a CSA-group by theorem 7. Moreover, each abelian subgroup of $G^{*}$ is either an $A$-module, or infinite cyclic. In both cases, they are faithful over $A$.

Lemma 7 The group $G^{*}$ has the following universal property with respect to the canonical embedding $i: G \hookrightarrow G^{*}$ : for any $A$-group $H$ and any partial A-homomorphism $f: G \longrightarrow H$ there exists a partial A-homomorphism $f^{*}: G^{*} \longrightarrow H$ such that $f=f^{*} \circ i$. 
Let $M$ be a maximal abelian subgroup of $G$, and $M \in \mathcal{C}$. Then $f(M)$ generates an abelian $A$-subgroup $N$ in $H$. Using the universal property of tensor completion for abelian groups, we can find a homomorphism $\psi_{M}$ : $M \otimes A \longrightarrow N$ such that $f=\psi_{M} \circ i_{M}$, where $i_{M}: M \hookrightarrow M \otimes A$ is the canonical embedding. By proposition 8, there exists $f^{*}: G^{*} \longrightarrow H$ with the property $f=f^{*} \circ i$.

Proof of the theorem. Let $G^{(n+1)}=\left(G^{(n)}\right)^{*}, G_{0}=G$, where $G^{*}$ is defined as above. Then the union $\bigcup_{n \in \omega} G^{(n)}=G \otimes A$ is the tensor $A$-completion of $G$. Indeed, $G \otimes A$ is a partial $A$-group as a union of partial $A$-groups, but an action of $A$ on $G \otimes A$ is defined everywhere: if $x \in G \otimes A$, then $x \in G^{(n)}$, and hence by lemma 5 the action of $A$ on $x$ is defined in $G^{(n+1)}$. So $G \otimes A$ is an $A$-group.

To prove that $G \otimes A$ is a tensor completion of $G$, we need to verify the corresponding universal property. Let $H$ be an $A$-group and $f: G \longrightarrow H$ a homomorphism. Using lemma 7 , we can extend $f$ to an $A$-homomorphism $f_{n}^{*}: G^{(n)} \longrightarrow H$ of partial $A$-groups. But then $f^{*}=\bigcup_{n<\omega} f_{n}$ is an $A$ homomorphism such that $f=f^{*} \circ i$, where $i$ is the canonical embedding $i: G \longrightarrow G \otimes A$.

Corollary 4 Let $G$ be a non-abelian $C S A$-group and $A \geq \mathbf{Z}$ a ring without additive elements of order 2. Then $G$ is faithful over $A$ iff each its abelian subgroup is faithful over $A$.

Corollary 5 Let $G$ be an $A$-faithful non-abelian $C S A$-group and $A$ a ring without additive elements of order 2. Then the tensor $A$-completion of $G$ is also a CSA-group.

Theorem 9 Let $G$ be a torsion-free $C S A$-group and $A$ a ring with a torsionfree additive group $A^{+}$. Then $G^{A}$ is a torsion-free CSA-group, and the canonical map $i: G \longrightarrow G^{A}$ is injective. In particular, this is true for a hyperbolic torsion-free group $G$, a group $G$ acting freely on a $\Lambda$-tree, and a universally free group $G$.

A corollary of the previous theorem and proposition 12 . 


\section{A-free groups}

In this section we investigate some basic properties of $A$-free groups. First of all we establish some properties of $F^{A}$ as a CSA-group, then introduce canonical forms of elements of $F^{A}$ and describe commuting elements and conjugate elements in $F^{A}$.

Let $A$ be a ring with a torsion-free additive group $A^{+}$, and $F_{A}(X)$ an $A$-free group with base $X$. From theorem 2 we know that $F_{A}(X)$ is the $A$-completion of the ordinary free group $F(X)=F$. The group $F$ is a CSAgroup, and all maximal abelian subgroups of $F$ are faithful over $A$ (because they are infinite cyclic), therefore the $A$-completion $F^{A}$ is the complete tensor extension of centralizers of $F$ (theorem 8 ), and we can apply results of sections 4 and 5 to $F^{A}$. In the case $A=\mathrm{Q}$ G.Baumslag obtained similar results for the group $F^{\mathbf{Q}}$.

Theorem 10 Let $F_{A}(X)$ be a non-abelian $A$-free group. Then

1. the canonical mapping $F \longrightarrow F_{A}(X)$ is injective;

2. $F_{A}(X)$ is a torsion-free group with conjugately separated maximal abelian subgroups;

3. $F_{A}(X)$ has no nonabelian solvable subgroups;

4. $F_{A}(X)$ has no nonabelian Baumslag - Solitar subgroups;

5. $F_{A}(X)$ has trivial center and is directly undecomposable

From theorem 8 and proposition 9.

By theorem 8, the group $F_{A}(X)$ is the union of the chain of subgroups:

$$
F=F^{(0)}<F^{(1)}<\cdots<F^{(n)}<\cdots
$$

where $F^{(n+1)}=F^{(n)}\left(C_{n}, A\right)$, and the set $C_{n}$ of centralizers in $F^{(n)}$ satisfies the condition (S), i.e. any centralizer from $C_{n}$ is infinite cyclic, no two of them are conjugate, and any cyclic centralizer in $F^{(n)}$ is conjugate to one from $C_{n}$. If $C(v)=\langle v\rangle \in C_{n}$, then the element $v$ is called a root element of level $n ; V_{n}$ is the set of all root elements of level $n$ such that only one 
generator of the subgroup $\langle v\rangle$ belongs to $V_{n} ; V=\cup_{n \in \omega} V_{n}$. The level function $\nu: F_{A}(X) \longrightarrow \omega$ is defined by the rule:

$$
\nu(g)=n \Longleftrightarrow g \in F^{(n)} \backslash F^{(n-1)},
$$

the number $\nu(g)$ is said to be the level of the element $g$.

Let us describe normal forms of elements of $F_{A}(X)$.

By section 4 , if $\nu(g)=n$, then $g$ can be written in reduced form:

$$
g=u_{1} v_{1}^{a_{1}} u_{2} v_{2}^{a_{2}} \cdots u_{m} v_{m}^{a_{m}} u_{m+1},
$$

where $a_{i} \in A \backslash \mathbf{Z}, v_{i} \in V_{n}, \nu\left(u_{i}\right)<n$, and if $v_{i}=v_{i+1}$, then $u_{i} \notin v_{i}^{\mathbf{Z}}$. Moreover, we can assume that the elements $u_{1}, \ldots, u_{m+1}$ are also in reduced form as elements of $F^{(n-1)}$. This leads us to an explicit reduced form of $g$. Similarly, following section 4, one can introduce canonical and semicanonical forms of elements and their explicit analogs.

Propostion 14 Let $g$ and $h$ be in reduced form (4):

$g=u_{1} v_{i_{1}}^{a_{1}} \cdots u_{m} v_{i_{m}}^{a_{m}} u_{m+1}, \quad h=w_{1} v_{j_{1}}^{b_{1}} \cdots w_{k} v_{i_{k}}^{b_{k}} w_{k+1}$.

Then $g=h$ if and only if

a) $m=k$ and $i_{s}=j_{s}, a_{s}=b_{s}+n_{s}$ for some $n_{s} \in \mathbf{Z}, s=1, \ldots, k$;

b) $u_{m+1}=v_{i_{m}}^{t_{m}} w_{m+1}, u_{m}=v_{i_{m-1}}^{t_{m-1}} w_{m} v_{i_{m}}^{-t_{m}-n_{m}}, \ldots, u_{1}=w_{1} v_{i_{1}}^{-t_{1}-n_{1}}$, where $n_{s}$ is as in 1$), t_{s} \in \mathbf{Z}, s=1, \ldots, m$.

Propostion 15 Suppose that $g$ and $h$ are in reduced form as in proposition 14 and $a_{s}=b_{s}$ for all $s$. Then $g=h$ iff $g$ can be obtained from $h$ by $a$ finite number of commutations of the type $v^{a} v^{t}=v^{t} v^{a}, a \in A, v \in V_{n}, t \in \mathbf{Z}$.

The following proposition gives us a description of commuting elements in $F_{A}(X)$ which is completely analogous to the case of ordinary free groups.

Propostion 16 Let $F_{A}(X)$ be a nonabelian A-free group. Then:

1. elements $x, y \in F_{A}(X)$ commute if $x=z^{a}, y=z^{b}$ for some $z \in F_{A}(X)$ and $a, b \in A$;

2. centralizers in $F_{A}(X)$ are exactly free $A$-modules of rank 1. 
$\square$ By theorem $8, F_{A}(X)$ is the complete tensor extension of centralizers by the ring $A$. According to this construction, if $g \in F_{A}(X)$ is an element of level $\alpha$, then by lemma $4 C(g) \simeq \mathbf{Z} \otimes_{\mathbf{z}} A \simeq A$, and the centralizer $C(g)$ is not extended on the next levels. Hence 2) is proved, and 1) follows from 2).

The reduced form (4) of an element $g$ is called cyclically reduced if any cyclic permutation of this form is also reduced.

Theorem 11 Let $g$ be a cyclically reduced element from the A-free group $F_{A}(X)$. Then

1. if $g$ is conjugate to an element $h$, then $h$ has the same level as $g$, and the level of the conjugating element is not greater than that of $g$;

2. if $g$ is conjugate to a cyclically reduced element $p_{1} \cdots p_{n}, n \geq 2$, of level $k$, then $g$ can be obtained by permuting $p_{1}, \ldots, p_{n}$ cyclically and then conjugating by a root element of level $k-1$.

Follows from lemma 2 from section 6 and the construction of the complete tensor extensions of centralizers of $F$.

\section{Open problems}

Question 1 Using extensions of centralizers, construct the A-completions of free solvable groups and groups of the type $F /[N, N]$ (these groups are not CSA-groups).

Question 2 Prove that the A-completion of a free nonabelian nilpotent (solvable) group can be non-nilpotent (non-solvable).

The affirmative answer to question 3 will show the necessity to define the notion of $A$-completion relatively to a variety of groups.

Question 3 Describe the tensor A-completion relatively to the varieties of nilpotent and solvable groups. 
Let us mention that Hall's $A$-completion of a nilpotent group could be different from that involved in question 4.

Finally we formulate some algorithmic problems for free $A$-groups over an effective [18 integral domain $A$ of characteristic 0 .

Question 4 Prove that in the A-free group $F^{A}$ the word problem and the conjugacy problem are algorithmically decidable.

Question 5 Is the Diophantine problem over an A-free group decidable?

Question 6 Is the universal theory of an A-free group decidable?

If the domain $A$ is residually $\mathbf{Z}$, then the answres to questions 5,6 and 7 are affirmative.

\section{References}

[1] Amaglobeli M.G. On commutativity of the functor of tensor completion with basic group operations. Omsk University, N7, 1992, p.1-11.

[2] Amaglobeli M.G., Remeslennikov V.N. Exponential groups: faithful Acompletion. Omsk University, N11, Omsk, 1993, p. 1-20 (in Russian).

[3] Bass H. Groups acting on non-archimedian trees. Arboreal group theory, 1991, p. 69-130.

[4] Baumslag G. Some aspects of groups with unique roots. Acta Math., 1960-, 104, p. 217-303.

[5] Baumslag G., Solitar D. Some two-generator one-relator non-Hopfian groups. Bull. Amer. Math. Soc. 68 (1962), p. 199-201.

[6] Ghys E., P. de la Harpe Sur les groupes hyperboliques d'apres Mikhael Gromov. Progress in Mathematics, v. 83, 1990.

[7] Hall P. Nilpotent groups. Canad. Math. Congress, Edmonton, 1957. 
[8] Hartley B. The residual nilpotence of wreath products. Proc. London Math. Soc., 20 (1970), 365-392.

[9] Lyndon R. Groups with parametric exponents. Trans. Amer. Math. Soc. - 1960 -v. 96 - p. 518-533.

[10] Magnus W., Karras A., Solitar D. Combinatorial group theory. Interscience publ., 1966, p. 1-444.

[11] Mal'cev A.I. Torsion free nilpotent groups. Izv. AN SSSR, Ser. Math. - 1949 - 13, N3, p. 201-212.

[12] Mal'cev A.I. On free solvable groups. Dokl. Ac. Nauk SSSR, 1960, v. 130, N3, p. 495-498.

[13] Myasnikov A.G. Centroid of a group and its links with endomorphisms and rings of scalars. Nova Journal of Algebra and Geometry, 1994 (to appear).

[14] Myasnikov A.G. Model-theoretic problems of algebra. Dissertation, Omsk, 1992.

[15] Myasnikov A.G., Remeslennikov V.N. Exponential groups I: foundations of the theory and tensor completion. Siberian Math. J., 1994, v.5.

[16] Myasnikov A.G., Remeslennikov V.N. Exponential groups: iterated tree extensions of centralizers. Omsk University, N18, Omsk, 1994, p. 1-30.

[17] Remeslennikov V.N. E-free groups. Siberian Math. J.- 1989- v.30, N6, p. $153-157$.

[18] Rabin M.O. Computable algebra, general theory and theory of computable fields. Trans. Amer. Math. Soc., 1960, 95, N2, p. 341-360. 